\title{
Research of Virtual Channels Scheduling Algorithm Based on Priority in Packet Telemetry System
}

\author{
TIAN Ye ${ }^{\mathrm{a}, \mathrm{b}}$, ZHANG Zi-jing $^{\mathrm{a}}$, ZHANG Yan-qin ${ }^{\mathrm{a}}$, XIAO Guang-yuan ${ }^{\mathrm{a}}$ \\ ${ }^{a}$ School of Information Science and Engineering, Shenyang Ligong University, Shenyang, China \\ ${ }^{b}$ State Key Laboratory of Rail Traffic Control and Safety, Beijing Jiaotong University, Beijing, China
}

\begin{abstract}
We make research on the virtual channels scheduling algorithm based on priority in packet telemetry system. Probability of occupying physical channel and packets losing rate of the virtual channel with the highest priority are considered, on condition that the packet arrival rate contribution is Poisson contribution. Theoretical analysis and simulation results show that when the running time of the scheduling module is long enough, probability of occupying physical channel by the virtual channel with the highest priority converge on a fixed value. Formulas of calculating packets losing rate are also given, which can offer reference to engineering design.
\end{abstract}

Index Terms: Virtual channels; scheduling algorithm; priority; packet telemetry

(C) 2012 Published by MECS Publisher. Selection and/or peer review under responsibility of the Research Association of Modern Education and Computer Science

\section{Introduction}

Consultive Committee for Space Data Systems has designed a group of space communication standards since it is established in 1982, which are now widely used in more than 250 countries and regions[1][2]. Among these standards, TM Space Data Link protocol is a data communication and transmission protocol, which is mainly used in packet telemetry system to transmit packet telemetry data from space to ground[3][4]. In order to better transmit those information, virtual channels scheduling mechanism is widely used in packet telemetry system.

Virtual Channels are a group of logic channels formed by dividing the physical channel by time slots. Each virtual channel transmits the packets information with the same or similar service demands, and the physical channel is shared by all these virtual channels. The algorithm used in virtual channels mechanism, namely, virtual channels scheduling algorithm, determines the sequence of occupying the physical channel by each virtual channel, which has a great effect on the transmission efficiency. In this paper, we study the virtual channels scheduling algorithm based on priority in packet telemetry system, and find an interesting conclusion: when the running time of the scheduling module is long enough, for the VC with the highest priority, both the probability of occupying the physical (namely, the VC with the highest priority is scheduled) and the packets losing rate converge on fixed values, which can provide reference for engineering design.

* Corresponding author.

E-mail address: tianyereal@ 126.com 


\section{Source Model and Division of Virtual Channels}

In this paper, we choose the packet telemetry source model, of which the physical channel is divided into 4 virtual channels to transfer all user data through the downlink [5]. The 4 virtual channels are shown as follows:

$\mathrm{VC1}$ : transmitting general engineering data, with the second higher priority.

VC2: transmitting important express data, with the highest priority.

VC3: transmitting download data, with the lowest priority.

VC4: producing idle frames. At any scheduling time point, if all the other VCs buffers are empty, an idle frame is generated in VC4 buffer and transmitted through the physical channel.

For $\mathrm{VCi}, i=1,2,3$, the packet arriving process is Poisson distribution with the parameter $\lambda_{i}$, and the packet length is a fixed value[6]. Let $l_{p i}$ be the Packet length, and $l_{m p}$ be the MPDU packet zone length of the frame, then each frame contains $N_{i}=l_{m p} / l_{p i}$ packets, which also means that a frame is generated and sent to the VCi buffer at the arriving time of the $m N_{i}$ packet, $m=1,2,3 \cdots$.

At each scheduling time point, the scheduling module choose the VC with the highest priority among the VCs whose frame buffers are not empty, and transmit the first frame in its buffer through the physical channel. If buffers of VC1, VC2, VC3 are all empty, an idle frame is generated in VC4 buffer and transmitted.

\section{Theoretical Analysis}

At the first scheduling time point, $\Delta t$, if the number of packets arriving in VC2 packets buffer is larger than $\mathrm{N}_{2}$, then there will be at least one frame in VC2 frames buffer, which means VC2 can occupy physical channel. So we have the probability of $\mathrm{VC} 2$ occupying physical channel at the time $\Delta t, P_{V C 2}{ }^{\prime}(1)$, as

$$
P_{V C 2}{ }^{\prime}(1)=P\left(A_{V C 2}(\Delta t) \geq N_{2}\right)=\sum_{n=N_{2}}^{\infty} \frac{\left(\lambda_{2} \Delta t\right)^{n} e^{-\lambda_{2} \Delta t}}{n !}
$$

where $A_{V C 2}(\Delta t)$ means the number of packets arriving in VC2 during the time interval $[0, \Delta t]$, whose value can be calculated as[7]:

$$
P\left(A_{V C 2}(\Delta t)=n\right)=\frac{\left(\lambda_{2} \Delta t\right)^{n} e^{-\lambda_{2} \Delta t}}{n !}
$$

Otherwise, there will be no frames in VC2 frames buffer, which means VC2 can not occupy physical channel. So we have the probability of VC2 not occupying physical channel at the time $\Delta t, P_{V C 2}{ }^{\prime}(0)$, as

$$
P_{V C 2}^{\prime}(0)=P\left(A_{V C 2}(\Delta t)<N_{2}\right)=\sum_{n=0}^{N_{2}-1} \frac{\left(\lambda_{2} \Delta t\right)^{n} e^{-\lambda_{2} \Delta t}}{n !}
$$

Let $M$ be the capacity of the VC2 packets buffer, probability of losing $d$ packets of VC2 at the time $\Delta t, P_{l V C 2}^{\prime}(d)$,can be expressed as:

$$
P_{l V C 2}^{\prime}(d)=\left\{\begin{array}{lc}
\sum_{n=0}^{M} \frac{e^{-\lambda_{2} \Delta t}\left(\lambda_{2} \Delta t\right)^{n}}{n !} & d=0 \\
\frac{e^{-\lambda_{2} \Delta t}\left(\lambda_{2} \Delta t\right)^{M+d}}{(M+d) !} & d=1,2, \cdots, \infty
\end{array}\right.
$$

After the first scheduling time point, probability of $r$ packets remained in VC2 packets buffer, ${ }_{R_{V C 2}}{ }^{\prime}(r)$, is: 


$$
\begin{aligned}
& P_{R_{V C 2}}(r)=\left\{\begin{array}{lc}
\frac{\left(\lambda_{2} \Delta t\right)^{N_{2}+r} e^{-\lambda_{2} \Delta t}}{\left(N_{2}+r\right) !}+\frac{\left(\lambda_{2} \Delta t\right)^{r} e^{-\lambda_{2} \Delta t}}{r !} & r=0,1, \cdots, M-N_{2}-1 \\
\sum_{i=M}^{\infty} \frac{\left(\lambda_{2} \Delta t\right)^{i} e^{-\lambda_{2} \Delta t}}{i !}+\frac{\left(\lambda_{2} \Delta t\right)^{M-N_{2}} e^{-\lambda_{2} \Delta t}}{\left(M-N_{2}\right) !} & r=M-N_{2} \\
\frac{\left(\lambda_{2} \Delta t\right)^{r} e^{-\lambda_{2} \Delta t}}{r !} & M-N_{2}<r<N_{2} \\
0 & \text { others }
\end{array}\right. \\
& P_{R_{V C 2}}(r)=\left\{\begin{array}{cc}
N_{2}<M<2 N_{2} ; & r=0,1, \cdots, N_{2}-1 \\
\frac{\left(\lambda_{2} \Delta t\right)^{N_{2}+r} e^{-\lambda_{2} \Delta t}}{\left(N_{2}+r\right) !}+\frac{\left(\lambda_{2} \Delta t\right)^{r} e^{-\lambda_{2} \Delta t}}{r !} & N_{2}-1<r<M-N_{2} \\
\sum_{i=M}^{\infty} \frac{\left(\lambda_{2} \Delta t\right)^{i} e^{-\lambda_{2} \Delta t}}{i !} & r=M-N_{2} \\
0 & \text { others }
\end{array}\right.
\end{aligned}
$$

if $M \geq 2 N_{2}$.

Similarly, at the kth scheduling time point, $k \Delta t, k=2,3 \cdots$, when the total number of the packets remained before the time $(k-1) \Delta t, R_{V C 2}((k-1) \Delta t)$, and the packets arriving during the time interval $[(k-1) \Delta t, k \Delta t]$, $A_{V C 2}((k-1) \Delta t, k \Delta t)$, is larger than or equal to $N_{2}$, there will be at least one frame in VC2 frames buffer. In this case, $\mathrm{VC} 2$ can be scheduled and the first frame in its buffer is transmitted through the physical channel, otherwise it cannot be scheduled. Thus we can get

$$
\begin{aligned}
P_{V C 2}{ }^{(k)}(1) & =P\left(\left[R_{V C 2}((k-1) \Delta t)+A_{V C 2}((k-1) \Delta t, k \Delta t)\right] \geq N_{2}\right) \\
& =\sum_{q=N_{2}}^{\infty} \sum_{i=0}^{q} P_{R_{V C 2}}{ }^{(k-1)}(i) P(A(\Delta t)=q-i) \\
P_{V C 2}{ }^{(k)}(0) & =P\left(\left[R_{V C 2}((k-1) \Delta t)+A_{V C 2}((k-1) \Delta t, k \Delta t)\right]<N_{2}\right) \\
& =\sum_{q=0}^{N_{2}-1} \sum_{i=0}^{q} P_{R_{V C 2}}{ }^{(k-1)}(i) P\left(A_{V C 2}(\Delta t)=q-i\right)
\end{aligned}
$$

where $P_{V C 2}{ }^{\left({ }^{k}\right)}(1)$ and $P_{V C 2}{ }^{(k)}(0)$ are the probability of VC2 can be scheduled and cannot be scheduled at the time $k \Delta t$, respectively.

Probability of losing $d$ packets of VC2 at the time $k \Delta t$ can be expressed as:

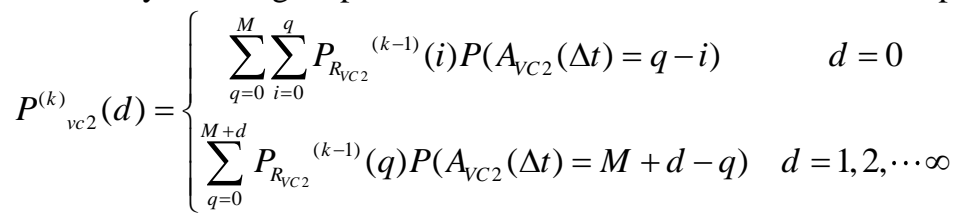

After the kth scheduling time point, probability of $r$ packets remained in VC2 packets buffer is 


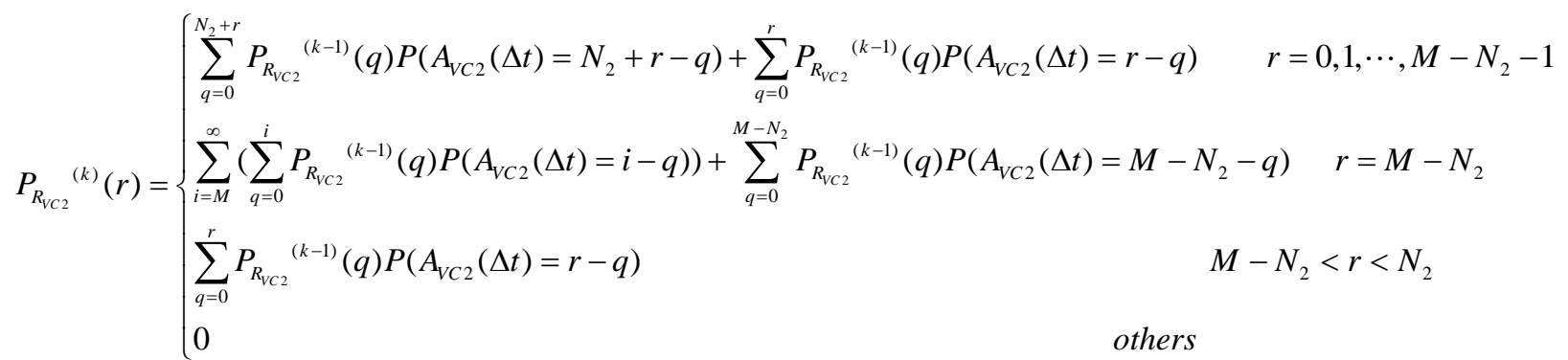

if $N_{2}<M<2 N_{2}$;

$P_{R_{V C 2}}{ }^{(k)}(r)= \begin{cases}\sum_{q=0}^{N_{2}+r} P_{R_{V C 2}}{ }^{(k-1)}(q) P\left(A_{V C 2}(\Delta t)=N_{2}+r-q\right)+\sum_{q=0}^{r} P_{R_{V C 2}}{ }^{(k-1)}(q) P\left(A_{V C 2}(\Delta t)=r-q\right) \quad r=0,1, \cdots, N_{2}-1 \\ \sum_{q=0}^{N_{2}+r} P_{R_{V C 2}{ }^{(k-1)}(q) P\left(A_{V C 2}(\Delta t)=N_{2}+r-q\right)} & N_{2}-1<r<M-N_{2} \\ \sum_{i=M}^{\infty}\left(\sum_{q=0}^{i} P_{R_{V C 2}}{ }^{(k-1)}(q) P\left(A_{V C 2}(\Delta t)=i-q\right)\right) & r=M-N_{2} \\ 0 & \text { others }\end{cases}$

if $M \geq 2 N_{2}$

\section{Simulation Results}

Simulation Parameters are set as follows:

(1) Source data rate of VC1, VC2 and VC3 are taken 10,10, 35 Mbps, respectively;

(2) The MPDU packet zone length of frame, ${ }_{m p}$, is $10000 \mathrm{bits}$;

(3) The packet length of VC1, VC2 and VC3 are 1000, 2000, 500bits, respectively;

(4) The average packet arrival rate of VC1, VC2 andVC3 are 10000, 5000, 70000/s, respectively;

(5) The total simulation time $\mathrm{T}$ is $0.8 \mathrm{~s}$.

Fig.1 illustrates the probability that VC2 is scheduled at different scheduling time points.

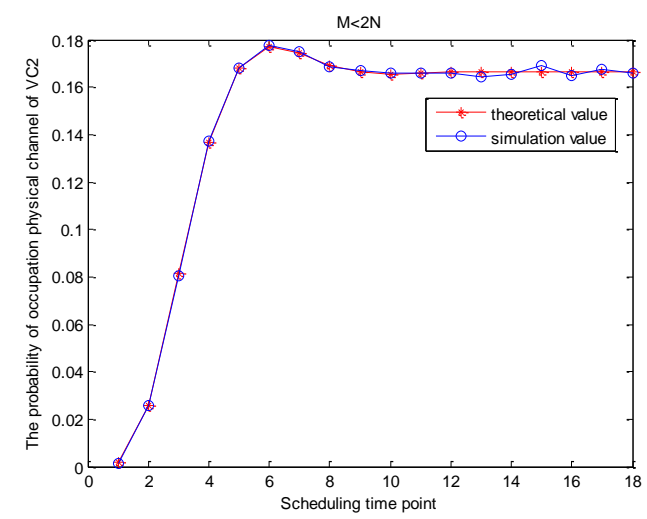

(a) $N<M<2 N$ 


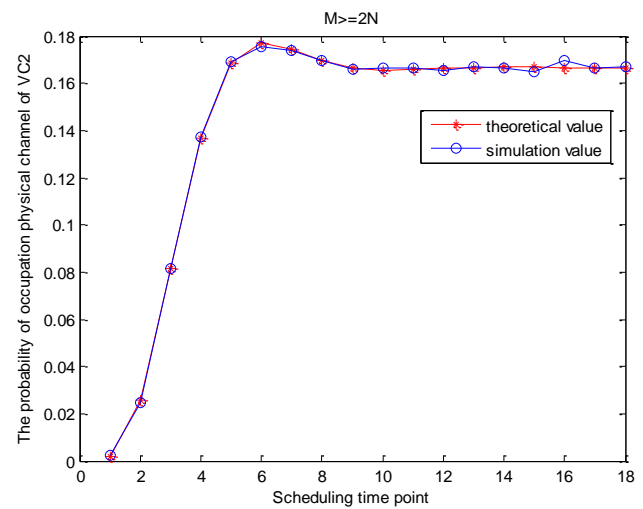

(b) $M \geq 2 N$

Fig.1 Probability that VC2 is scheduled at different scheduling time points

From the simulation results we can see that with the increasing of the running time of the scheduling module, the probability that VC2 can be scheduled converge on a fixed value, and the simulation curves almost completely coincide with the theoretical curves, which proves the correctness of the theoretical analysis in section III.

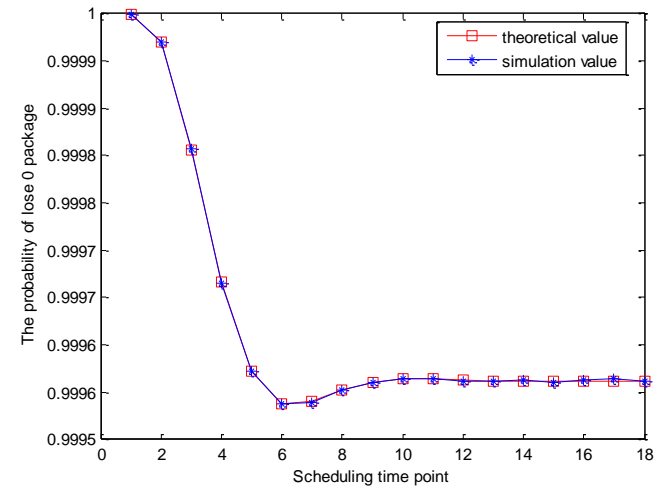

(a) Probability of losing 0 packet

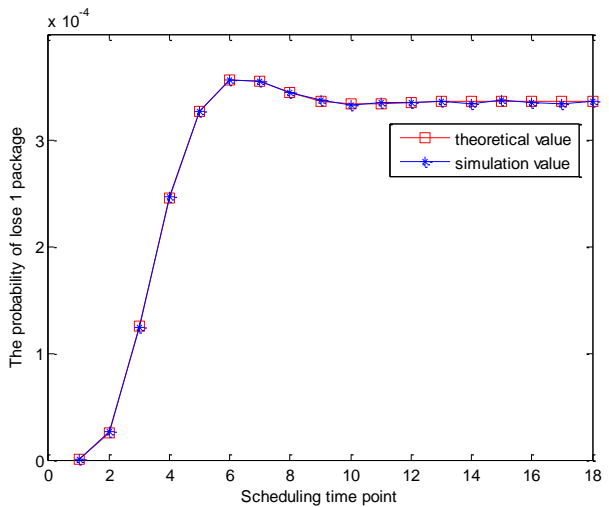

(b) Probability of losing 1 packet 


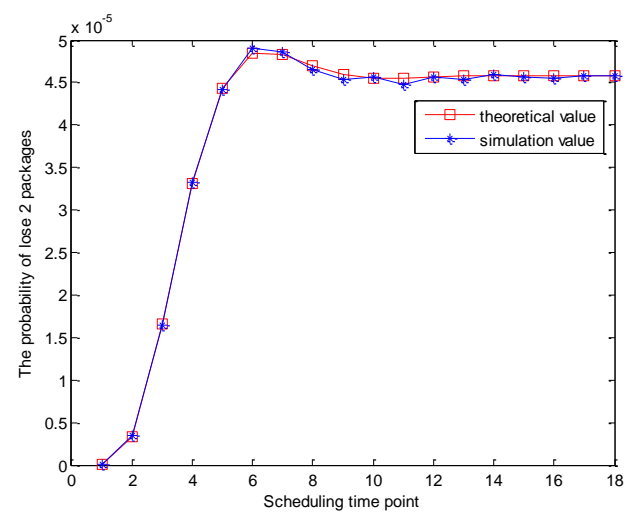

(c) Probability of losing 2 packets

Fig.2 Packets losing rate of VC2 at different scheduling time points

Fig.2 illustrates the curve of the theoretical value and simulation value of the packets losing rate of VC2 at different scheduling time points when the packet buffer capacity is $M(M=8)$, which proves the correctness of equation(8). In practical application, one can design the value of $M$ according to the desired packets losing rate.

\section{Conclusion}

We study the scheduling algorithm based on priority in packet telemetry system. Through severe reasoning and simulation experiment, we find that when the running time of the scheduling module is long enough, the probability that the VC with the highest priority can be scheduled converge on a fixed value. We also propose the method to calculate packets losing rating of the $\mathrm{VC}$ with the highest priority, which can offer reference to engineering design.

\section{Acknowledgements}

Support by the State Key Laboratory of Rail Traffic Control and Safety (RCS2009K008), Beijing Jiaotong University.

\section{References}

[1] Zhao He-ping, Li Ning-ning. Implementation of CCSDS standard in military space mission[J]. Spacecraft Engineering, 2007, 16(4):78-82.(in Chinese)

[2] CCSDS. CCSDS missions. http: //public. ccsds.org/ implementations/ missions.aspx. 2007

[3] CCSDS. Space packet protocol[S]. CCSDS 133.0-B-1, Blue Book, September 2003.

[4] CCSDS. TM space data link protocol[S]. CCSDS 132.0-B-1, Blue Book, September 2003.

[5] Gu Ying-qi, Tan Wei-chi. CCSDS downlink virtual channel schedule and performance analysis[J]. Chinese Space Science and Technology, 2001,21(3):29-35.(in Chinese)

[6] Ba Yong, Zhang Nai-tong. Analysis of CCSDS protocol and space data system[D]. Harbin: Harbin Institute of Technology, 2000(in Chinese)

[7] Mao Yong-cai, Hu Qi-ying. Stochastic Process[M]. Xi'an : Xian Electronics Science and Technology University Press. 2006:39-45(in Chinese) 